\title{
Delirium in the intensive care unit: Assessment, treatment and implications for practice
}

Nancy McNamara RCpN, BN, Intensive Care Nurse, Tauranga Hospital, New Zealand

E-mail: gandnmcn@xtra.co.nz

Key words: Intensive care unit (ICU * delirium * CAM-ICU *assessment $*$ stressors $*$ Haliperidol

\section{SUMMARY \\ * Delirium in ICU is a complication that affects survival and long-term quality of life, especially in older adults. \\ * A validated assessment tool such as Dr Ely's CAM-ICU helps to detect early signs and symptoms. \\ * Interventions such as reducing environmental stressors, effective pharmacological and non-pharmacological pain management, and careful monitoring, help to minimise the effects of delirium. \\ * Ongoing medical and nursing research is necessary to further understand and manage delirium.}

\section{INTRODUCTION}

The advanced technologies and therapies used in an intensive care unit (ICU) have the ability to preserve and save lives but they may also dehumanise the environment. An ICU can be a frightening place, because the way people normally communicate, interpret and orientate themselves is disrupted or disabled. Sight, touch, hearing and communication are impaired by changes in physiology and drugs, which may also distort reality As patients respond to fear, pain, sleep deprivation, medication and isolation, they become confused and may have perceptual disturbances such as hallucinations or delusions (Powell, 2002).

In this article I will define ICU delirium and discuss the incidence and clinical outcomes of it. I will then explore types of delirium and discuss risk factors, both predisposing and precipitating/environment. I will then look at the assessment for delirium and show some clinical guidelines for treatment. The role of the ICU nurse will be discussed next and how they can help reduce environmental stressors. The role of families will also be explored and the implications for further practice discussed.

\section{DEFINITION}

The word delirium is derived from the Latin 'delirare' and means 'to act crazy' or to 'run off the tracks' (Roberts, 2001). The Diagnostic and Statistical Manual of Mental Disorders (4th ed.) and the International Classification of Diseases (10th revision) both define delirium as a disturbance of consciousness and attention with a change in cognition or perceptual disturbance. Symptoms develop with a rapid onset and delirium has an underlying medical cause (McGuire et al., 2000).

\section{INCIDENCE OF DELIRIUM AND ASSOCIATED CLINICAL OUTCOMES}

In the United States of America (USA), 55,000 people are treated in ICUs daily. Of that number, nearly 40,000 are expected to develop delirium (Pasley, 2004). Dr Wesley Ely is the associate professor of medicine at Vanderbilt University School of Medicine. He is also the associate director for the Geriatric Research and Education Clinical Centre (GRECC). In 2001, Ely et al. (2004) began a fiveyear cohort investigation of cognitive impairment in ICU patients. They focused on delirium and neuropsychological impairment in older survivors of critical illness.

The team set out to determine if delirium was an independent predictor of clinical outcomes, including six-month mortality and length of stay among ICU patients receiving mechanical ventilation. The research was a prospective cohort study involving 275 mechanically ventilated patients. The participants were admitted to adult medical and coronary ICUs in an American universitybased, 631-bed medical centre. Patients were followed up for the development of delirium over 2,158 ICU days using the Confusion Assessment Method for the ICU (CAM-ICU) and the Richmond Agitation-Sedation Scale. Patients' baseline demographic and clinical variables were assessed using Wilcox rank sum tests for continuous variables and Fisher exact tests for comparing proportions.

During the study, 51 participants never woke up from coma. The remaining 224 patients were divided into two groups according to whether they developed delirium. In the six-month follow-up period, $34 \%(63 / 183)$ of the patients in the delirium group died versus $15 \%(6 / 41)$ of the patients in the non-delirium group ( $p=.03)$. A time-dependant, multivariable Cox proportional hazard model was used to adjust for all 11 of the covariates in the study. The results revealed that delirium was associated with a three times higher risk of dying by six months. A similar analysis, which considered the duration of delirium, also found that each additional day an ICU patient spent in delirium was associated with a $10 \%$ increased risk of dying $(p=.03)$.

Further research by Ely et al. (2001) revealed that those who developed delirium spent a median of 10 days longer in hospital overall and their risk of remaining in the wards after ICU discharge was $60 \%$ higher than those who never developed delirium. The 
research concluded that delirium was an independent predictor of higher six-month mortality and total hospital stay, even after adjusting for relevant covariates including coma, sedatives and analgesics in patients receiving mechanical ventilation.

Milbrandt et al. (2004) then researched the costs involved with increased hospital stays. Costs were determined from individual ledger-level patient charges using cost centre specific cost-tocharge ratios and were reported in 2001 in US dollars from the hospital perspective. Baseline characteristics were compared between patients who had experienced delirium and those who never experienced it, using Fisher extract Wilcox on rank sum tests or Student's t-tests as appropriate.

Using the cumulative delirium severity index, the research showed that greater severity and duration of delirium was associated with greater costs. After adjustments for age, co-morbidity, severity of illness and degree of organ dysfunction, delirium was associated with $39 \%$ higher ICU costs $(p=.003)$ and 31\% higher total hospital costs $(p=0.4)$.

Further research by McNicholl et al. (2003) revealed that, of those who survive, at least one in three will experience long-term cognitive impairment. Clinically significant depression may occur in as many as $30 \%$ of ICU survivors and between $15 \%$ and $40 \%$ of these patients experience symptoms of post-traumatic stress disorder (PTSD).

\section{TYPES OF DELIRIUM}

There are three subtypes of delirium: hypoactive, hyperactive or a mixture of both (Truman \& Ely, 2003). Hypoactive delirium is the most common subtype experienced by ICU patients and is often referred to as the 'quiet' type. Symptoms of this subtype are withdrawal, lethargy, apathy and occasionally a total lack of responsiveness (Justic, 2000). This subtype is associated with longer hospital stays and higher mortality rates.

Hyperactive delirium, the second subtype, is easily recognisable because of the extreme agitation, excessive activity and disruptive behaviour patients often display. Patients with this subtype will often try to pull out catheters and tubes, get out of bed, and may hit out at staff. Patients with this subtype are often given higher doses of sedatives to help maintain their physical safety (Truman \& Ely, 2003).

Patients with the mixed subtype show symptoms of both hypoactive and hyperactive delirium. This subtype is also common in ICU and may be particularly hard for families and staff to understand and cope with. Drowsy lethargic patients can be lucid for a period and then become loud and aggressive the next time they are visited or assessed (Justic, 2000).

\section{RISK FACTORS}

There is no single cause for the development of delirium. Risk factors, however, may be divided into two categories: predisposing and precipitating. Predisposing factors are present at the time of admission and reflect the baseline vulnerability of a patient. Precipitating factors are noxious insults or hospital-related factors (Schuurmans et al., 2001).

\section{Predisposing Factors}

Age is the single most important predisposing factor for delirium. Older adults (over 65) have a greater risk of developing delirium simply because of the physiological effects of aging, such as a reduced capacity for homeostatic regulation and altered pharmacokinetics. Older adults often have co-morbidities for which they take a number of daily medications, thus increasing the likelihood of polypharmacy and drug interaction (Marshall \& Soucy, 2003). Dementia is another major predisposing factor. A study in an American medical centre by McNicholl et al. (2003), which included 185 ICU patients aged 65 and over, revealed that $40 \%$ of patients with dementia would develop delirium by the end of the post-ICU period, even after controlling for co-morbidities, baseline functional status, severity of illness and invasive procedures $(\mathrm{p}=<0.05)$.

Existing Alzheimer's disease and a history of alcohol or substance abuse, severe illness and dehydration are also major predisposing factors for the development of delirium in older adults.

\section{Precipitating/Environmental Factors}

Precipitating risk factors include: hypoxia underlying chronic systemic illness, metabolic or haemodynamic instability, electrolyte imbalances, severe infections and brain tumours (Roberts, 2001). Medications are a subclass and alone place patients at a higher risk of delirium. In ICU, more than $95 \%$ of patients are regularly given sedatives, opiates, drugs with anticholinergic properties and psychoactive drugs (Truman \& Ely, 2003). These drugs affect the balance of neurotransmitters in the central nervous system, which in turn affects mood, behaviour and cognitive function. Withdrawal from benzodiazepines, opiates and sedation drugs in ICU is a major precipitator of delirium.

Environmental stressors within ICU may also precipitate the onset of delirium (Truman \& Ely, 2003). 'White noise' or the constant sound from ventilators, machinery and alarms, and 'white light', the constant artificial light source, may further add to sensory overload for the patient. Constant interruption by nursing and medical staff may also be a stressor.

Meyer et al. (1994) performed a study in a 720-bed university teaching hospital in Province, Rhode Island. Light and sound levels were monitored continuously for seven days in areas that were considered to represent a range of care intensity. Patient interruptions were sampled for 24 hours. The study revealed that light levels showed normal rhythmicity with peak levels occurring during daylight hours. Peak sound levels, however, were extremely high in all areas, with peak levels averaging 82.6 decibels. The United States Environmental Protection Agency (1974) recommends that noise levels in hospital should not exceed 45 decibels in the day or 35 decibels at night. Interruptions were found in the study to occur at least hourly, which did not allow time for condensed sleep. Even the slightest interruption, like moving IV lines, was enough to disrupt the patient and affect the normal circadian sleep cycles.

Pain has been reported by patients as the greatest stressor in ICU. Epstein and Breslow (1999) state that unrelieved pain evokes a stress response characterised by tachycardia, increased myocardial oxygen consumption, hypercoagulability, immunosuppression and persistent catabolism. It may also contribute to inadequate sleep and agitation. Research by Todres et al. (2000), which used a phenomenological approach and focused on client experiences, raised the question of whether it is too painful for patients to be lightly sedated. A nurse who had been an ICU patient on three occasions spoke of constant pain caused by intubation, being turned, suctioning, coughing, gastro intestinal disturbances caused by fluid management, restricted movement and physiotherapy. 


\section{*Delirium in the intensive care unit: Assessment, treatment and implications for practice *}

Communication is often impossible for ICU patients because of the presence of endotracheal or tracheostomy tubes. Non-verbal attempts such as lip reading and writing are often unsuccessful and may leave the patient feeling frustrated and insecure. A qualitative study in the United Kingdom using non-participant observation studied 16 nurses in an adult ICU over a three-week period. Each nurse was assessed for four hours. Results showed that nurses communicated with their patients for a mean of 3-5 minutes (Elliott \& Wright, 1999). It is even more difficult for patients who do not understand the dominant language (Roberts, 2001) - the experience of being in ICU for these patients may be even more frightening.

Different cultures view health in different ways and it is possible that the highly technical environment in ICU may clash with cultures more familiar with folk medicines, natural remedies, rituals and religious healing (Justic, 2000).

\section{ASSESSMENT FOR DELIRIUM}

Intensive care nurses with responsibility for 24-hour care at the bedside are usually the first to identify changes in patient behaviour and mental status. Hypoactive delirium, however, goes unrecognised in $66 \%$ to $84 \%$ of patients (Marshall \& Soucy, 2003). Many authors (McNicholl et al., 2003; Truman \& Ely 2003; Ely et al., 2001) agree that symptoms are best identified using a formal assessment tool. However, a recent study of health care professionals revealed that, although $92 \%$ agreed that delirium was a serious concern in $\mathrm{ICU}$, the tools used to assess this condition were designed to assess sedation rather than delirium (Tanios et al., 2004).

\section{The CAM-ICU}

Dr Ely's confusion assessment method CAM-ICU was designed especially to diagnose delirium in critically ill non-verbal patients. A prospective cohort study involving 38 adult patients in an American university-based medical centre was undertaken by Ely et al. (2001) to test the reliability and validity of the tool. A total of 293 daily paired evaluations were completed. The results showed that two critical care nurses and one intensivist using the CAMICU demonstrated high interrater reliability for their ratings with Kappa statistics of $0.84,0.79$ and 0.95 respectively $(p=.001)$. The research concluded that the CAM-ICU could be a reliable instrument for clinical and research purposes to diagnose and monitor delirium.

The study was repeated by the researchers later in 2001 with a larger sample of 111 consecutive patients who were mechanically ventilated. A total of 471 daily paired evaluations were collected in this study and high interrater reliability was noted with Kappa statistics of 0.96 . Delirium was diagnosed in $87 \%$ of patients with an average onset on the second day and a mean duration of 4.2 1.7 days. The research concluded that the CAM-ICU was rapid, valid and reliable.

\section{CLINICAL GUIDELINES FOR TREATMENT}

The first step in pharmacological treatment of delirium is to ensure that none of the patient's current medications are adding to the delirium. Many drugs, such as benzodiazepines and narcotics, often used to treat confusion may actually exacerbate the problem (Truman \& Ely, 2003).

At present, no drugs have been approved by the Food and Drug
Administration for the treatment of delirium (Truman \& Ely, 2003). The Society of Critical Care Medicine (SCCM) and the American Psychiatric Association (APA) have developed their own guidelines for clinical practice.

\section{APA guidelines for clinical practice \\ * Immediate intervention for urgent medical conditions; \\ * Identification and intervention of delirium; \\ * Means to ensure safety; \\ * Steps to improve a patient's functioning including altering the environment and providing emotional support (Marshall \& Soucy, 2003).}

\section{SCCM recommendations}

* Routine assessment for the presence of delirium. The CAM-ICU is noted as a promising tool

* The use of Haliperidol for treatment of delirium in critically ill patients

* Monitoring patients for electrographic changes while receiving Haliperidol

* Sleep promotion including optimisation of the environment and non-pharmacologic methods to promote relaxation with adjunctive use of hypnotics.

The SCCM also recommends the use of Dr Ely's delirium assessment tool (Jacobi et al., 2002).

\section{ROLE OF THE ICU NURSE}

Nursing management of delirium begins with identifying those patients at risk and frequently assessing for symptoms of delirium using a validated assessment tool. Interventions are then aimed at maintaining safety and comfort, re-orientating patients and reducing environmental stressors.

Patient safety is the first consideration. A study in the UK of 45 adult patients who spent a minimum of three days in ICU showed that their overwhelming need was to feel safe (Adam \& Forest, 1999). For a patient with signs of hyperactive delirium in a delusional state, maintaining their safety may be a major concern. Constant supervision and reassurance is necessary. All lines must be secured firmly. Catheters and endotracheal tubes must also remain secure to avoid self-extubation. Physical restraints are used in extreme cases but their use is recommended only as a last resort (Justic, 2000).

Constantly re-orientating patients, by explaining where they are, why they are there and what day it is, helps to minimise their fear and confusion. Putting a clock or a calendar in the room and asking family to bring in possessions such as photos may help the patient and increase feelings of safety (Adam \& Forest, 1999). Assuring the patient that their reactions and responses are normal and expected may also provide relief.

\section{Reducing environmental stressors}

ICU nurses can reduce alarm noise by setting realistic parameters and turning off unnecessary equipment such as suction apparatus. Radios may be turned on quietly and families may be asked to bring in favourite tapes or CDs. Medical staff and members of the multidisciplinary team can be steered away from the bedside to discuss progress and treatment (Litton, 2003).

Overnight, nursing interventions can be organised to ensure the least disruption. Blood sampling, medications and analgesia can 
be given before the patient is settled for the night. Turning off or dimming electric lights will also help to maintain the circadian cycle. It is often possible for ICU nurses to use natural lighting by elevating patients beds and facing them toward windows (Roberts, 2001).

\section{Pain}

Unless the patients themselves confirm that they are pain-free, nurses should assume that they have pain. Constant assessment and monitoring of pain levels is necessary. Suitable analgesics should be administered every two to four hours or by continuous infusion (Litton, 2003). Given a discretionary range for analgesia, ICU nurses are often able to titrate the dose depending on the patients' degree of pain. All interventions should maximise the patients' comfort.

\section{Communication}

Communication with a delirious patient should be brief and basic and be repeated frequently in a calm voice. All procedures should be explained before they start and the use of medical terminology should be avoided (Roberts, 2001). It is important to have an interpreter or family member present as often as possible if communication is difficult due to language differences. Boards or pictures may also be used for patients who are not able to communicate verbally.

$\mathrm{ICU}$ nurses can also convey compassion and empathy with attentiveness and touch to enhance communication when a patient cannot communicate. It may in fact reassure the patient that the nurse is focused on them and not the procedure or surrounding technology (Hewitt, 2002).

\section{INCLUDING FAMILIES}

One of the most common complaints from families of critically ill patients is that of being uninformed; not knowing whether their intervention was helpful or not, not knowing whether their loved one would ever fully recover from the delirium and not knowing what to expect on a daily basis (Hartwick, 2003). Educating family members about delirium, how it may manifest, what is expected and including them in the patient's plan of care are essential. It is the family that, in many cases, will provide ongoing care and support after discharge. One of the long-term effects of delirium is that patients have a greater ongoing need for post-discharge community support and services or an increased need for long-term placement (Truman \& Ely, 2003).

\section{IMPLICATIONS FOR FURTHER PRACTICE}

A constant theme throughout this study has been that delirium goes unnoticed by ICU nurses and predisposing factors for delirium are not recognised at admission. If the most effective way of managing delirium is to avoid its development or to minimise its severity then I propose education and a validated assessment tool is needed for nursing staff. The introduction of training programmes could include new staff orientating to ICU. Research by Ely et al. (2001) demonstrated that, with education and a validated assessment tool, $87 \%$ of patients were identified as developing the signs and symptoms of delirium. The concept of training and the introduction of an assessment tool for delirium in ICU would possibly be met with resistance from staff due to issues of understaffing, time constraints and heavy workloads. The assessment tool may simply be seen as more paperwork to add to the huge amount of documentation already required by ICU nurses, and time and distance may be issues for many nurses regarding education programmes. Education, however, could be incorporated into new staff orientation programmes.

Education for families could also help to reassure them that delirium is transient and a relatively common development. Resources could be developed in the form of a pamphlet.

With regard to environmental stressors, consideration in the structural design of any new or renovated ICUs should be considered. Natural light should be maximised and new technology in building materials, such as walls that may help filter sound, should be utilised.

Provision should be made for post-discharge follow-up both in hospital and in the community to assess ongoing needs and concerns related to the critical illness. The follow-up process also provides a significant opportunity for ongoing post-discharge research. This research is necessary to further investigate the longterm outcomes of ICU delirium and the effect of intervention strategies.

\section{CONCLUSION}

Research has proven that the development of ICU delirium is a serious complication especially in the elderly. Early recognition, assessment and prompt treatment, both pharmacological and nonpharmacological, is the recommended management strategy. ICU nurses who provide 24-hour care at the bedside have the ability to significantly impact on their patients' short and long-term experience of critical illness.

\section{REFERENCES}

Adam S \& Forest S. (1999) ABC of intensive care: Other supportive care. British Medical Journal 319 (7203), 175-178.

Elliott R \& Wright L. (1999) Verbal communication: what do critical care nurses say to their unconscious or sedated patients? Journal of Advanced Nursing 29 (6), 1412-1420.

Ely EW, Gautam S, Margolin R, Francis J, May L \& Speroff T, Truman B, Dittus R, Bernard R \& Inouye SK. (2001) The impact of delirium in the intensive care unit on hospital length of stay. Intensive Care Medicine 27 (12), 1892-1900.

Ely EW, Shintani A, Truman B, Speroff T, Gordon SM, Harrell FE, Inouye SK, Bernard GR \& Dittus RS. (2004) Caring for the critically ill patient. Delirium as a predictor of mortality in mechanically ventilated patients in the intensive care unit. Journal of the American Medical Association 291 (14), 1753-1762.

Epstein J \& Breslow MJ. (1999) The stress response of critical illness. Critical Care Clinician 15 (1), 17-33.

Hartwick S. (2003) ICU delirium: A case study. Critical Care Nursing Quarterly 26 (3), 221-224.

Hewitt J. (2002) Psycho-affective disorder in intensive care units: a review. Journal of Clinical Nursing 11 (5), 575-584.

Jacobi J, Fraser GL, Coursin DB, Riker RR, Fontaine D, Wittbrodt ET, Chalfin DB, Masica MF, Bjerke HS, Coplin WM, Crippen DW, Fuchs BD, Kelleher RM, Marik PE, Nasraway SA, Murray MJ, Peruzzi WT \& Lumb PD. (2002) Clinical practice guidelines for the sustained use of sedatives and analgesics in the critically ill adult. Critical Care Medicine 30 (1), 119-141.

Justic M. (2000) Does 'ICU psychosis' really exist? Critical Care Nurse 20 (3), 28-39. 
Litton KA. (2003) Delirium in the critical care patient: what the professional staff need to know. Critical Care Nursing Quarterly 26 (3), 208-213.

Marshall MC \& Soucy MD. (2003) Delirium in the intensive care unit. Critical Care Nursing Quarterly 26 (3), 172-178.

McGuire BE, Basten CJ, Ryan CJ \& Gallagher J. (2000) Intensive care unit syndrome: A serious misnomer. Archives of Internal Medicine 160 (7), 906-909

McNicholl L, Pisani MA, Zhang Y, Ely EW, Siegel MD \& Inouye SK. (2003) Delirium in the intensive care unit: occurrence and clinical course in older patients. Journal of the American Geriatrics Society 51 (5), 591-598.

Meyer TJ, Eveloff SE, Bauer MS, Schwartz WA, Hill NS \& Millman RP. (1994) Adverse environmental conditions in the respiratory and medical ICU settings. Chest 105 (4), 1211-1216.

Milbrandt EB, Deppen S, Harrison PL, Shintani AK, Speroff T, Stiles RA, Truman B, Bernard GR, Dittus RS \& Ely EW. (2004) Costs associated with delirium in mechanically ventilated patients. Critical Care Medicine 32 (4), 955-962.

Pasley J. (2004) Delirium in ICU patients: a predictor of mortality. The Reporter [online]. Available at: http://www.mc.vanderbilt.edu/reporter/ ?ID=3207 [Accessed 11 May 2004].
Powell J. (2002) Caring for patients after an ICU admission. Kai Tiaki: Nursing in New Zealand 8 (7), 24-25.

Roberts BL. (2001) Managing delirium in adult intensive care patients. Critical Care Nurse 21 (1), 48-55.

Schuurmans MJ, Duursma SA \& Shortridge-Baggett LM. (2001) Early recognition of delirium: review of the literature. Journal of Clinical Nursing 10 (6), 721-729.

Tanios MA, Epstein SK \& Teres D. (2004) Are we ready to monitor delirium in the intensive care unit? Critical Care Medicine 32 (1), 295-296.

Todres L, Fulbrook P \& Albarran J. (2000) On the receiving end: a hermeneutic-phenomenological analysis of a patient's struggle to cope while going through intensive care. Nursing in Critical Care 5 (6), 277-287.

Truman BE \& Ely WE. (2003) Monitoring delirium in critically ill patients: Using the confusion assessment method for the intensive care unit. Critical Care Nurse 23 (2), 25-36.

United States Environmental Protection Agency (1974). Information on levels of environmental noise requisite to protect public health and welfare with an adequate margin of safety. Washington, DC: Government Printing Office. 